\title{
Degrees and rationality of characters in the principal block of $A_{n}$
}

\author{
Eugenio Giannelli And Elena Meini
}

\begin{abstract}
Given two primes $p$ and $q$, we study degrees and rationality of irreducible characters in the principal $p$-block of $\mathfrak{S}_{n}$ and $\mathfrak{A}_{n}$, the symmetric and alternating groups. In particular, we show that such a block always admits an irreducible character of degree divisible by $q$. This extends and generalizes a recent result of Giannelli-Malle-Vallejo.
\end{abstract}

Mathematics Subject Classification. 20C30.

Keywords. Symmetric groups, Alternating groups, Principal block.

1. Introduction. Let $p$ be an odd prime. In a recent paper [1], the authors characterized finite groups whose principal $p$-block consists only of irreducible characters of odd degree. This result relied on the classification of finite simple groups. More precisely, it was shown that the principal $p$-block of every nonabelian finite simple group (with the sole exception of $M_{22}$ at the prime $p=7$ ) admits an irreducible character $\chi$ of even degree. In the case of alternating groups, [1, Theorem C] shows that a more general statement holds.

Theorem 1.1. Let $n \geq 5$, let $G$ be either $\mathfrak{S}_{n}$ or $\mathfrak{A}_{n}$, and let $p$ and $q$ be distinct primes with $q<p \leq n$. Then the principal p-block of $G$ contains an irreducible character of degree divisible by $q$.

Results that take into consideration two distinct primes are not frequent in representation theory of finite groups. Recently, given two distinct primes $p$ and $q$, the $q$-divisibility of the degrees of irreducible characters in the principal $p$-block of a finite group has been studied in [4]. There the authors propose a generalization of the famous Brauer height zero conjecture for the principal block and use Theorem 1.1 to prove one of the main results of the article [4, Theorem 5.1].

The main aim of this short note is to strengthen Theorem 1.1 by removing the hypotheses on the two primes $p$ and $q$. 
Theorem A. Let $n \geq 5$, let $p, q \leq n$ be distinct primes and let $G$ be either $\mathfrak{S}_{n}$ or $\mathfrak{A}_{n}$. Then the principal p-block of $G$ contains an irreducible character of degree divisible by $q$.

We remark that Theorem A provides an answer to a question raised in [1] at the end of Section 3.

In the second part of the paper, we ask ourselves whether the irreducible character identified by Theorem A can be chosen to be rational valued. The question has a trivial positive answer for $\mathfrak{S}_{n}$. For this reason, we focus our attention on alternating groups. We manage to prove the following complete classification.

Theorem B. Let $n \geq 5$, let $p, q \leq n$ be distinct primes. The principal $p$-block of $\mathfrak{A}_{n}$ contains an irreducible and rational valued character of degree divisible by $q$ unless:

(i) $n=p=2 q-1$, or (ii) $n=8, p=7$, and $q=3$, or $($ iii $) n=6, p=5$, and $q=2$.

2. Preliminaries. We recall some basic facts on the algebraic and combinatorial theory of representations of $\mathfrak{S}_{n}$. Our main references are [2] and [5]. The irreducible characters of $\mathfrak{S}_{n}$ are naturally labelled by partitions of $n$. Given a partition $\lambda=\left(\lambda_{1}, \ldots, \lambda_{z}\right)$ of $n$, we denote by $\chi^{\lambda}$ the corresponding irreducible character, and by $[\lambda]$ the associated Young diagram. We recall that

$$
[\lambda]=\left\{(i, j) \in \mathbb{N} \times \mathbb{N} \mid 1 \leq i \leq z, 1 \leq j \leq \lambda_{i}\right\},
$$

and that every element $(r, c) \in[\lambda]$ is called a node of $[\lambda]$. We denote by $H_{r, c}(\lambda)$ the hook associated to the node $(r, c)$, and we let $h_{r, c}(\lambda)=\left|H_{r, c}(\lambda)\right|$ be the length of the hook $H_{r, c}(\lambda)$. We recall that $H_{r, c}(\lambda)$ is the subset of $[\lambda]$ defined by

$$
H_{(r, c)}(\lambda)=\{(r, y) \in[\lambda] \mid y \geq c\} \cup\{(x, c) \in[\lambda] \mid x \geq r\} .
$$

Given integers $t$ and $r$, we let $\mathcal{H}_{r}^{t}(\lambda)=\left\{(r, c) \in[\lambda] \mid t\right.$ divides $\left.h_{r, c}\right\}$. Similarly we let $\mathcal{H}^{t}(\lambda)=\bigcup_{r=1}^{z} \mathcal{H}_{r}^{t}(\lambda)$ be the set of all nodes in $[\lambda]$ whose associated hook has length divisible by $t$. A partition $\gamma$ is a $t$-core if $\mathcal{H}^{t}(\gamma)=\emptyset$. Given a partition $\lambda$ of $n$, the $t$-core of $\lambda$ is the partition obtained from $\lambda$ by successively removing $t$-hooks. In this article, we denote this by $C_{t}(\lambda)$.

Given a prime $p$, we know from $[2,6.1 .21]$ that $\chi^{\lambda}$ and $\chi^{\mu}$ lie in the same $p$ block of $\mathfrak{S}_{n}$ if and only if $C_{p}(\lambda)=C_{p}(\mu)$. In particular, $\chi^{\lambda}$ lies in the principal p-block if and only if $C_{p}(\lambda)=C_{p}((n))$ is a one-part partition.

A complete study of the factorization into primes of the degree of irreducible characters of $\mathfrak{S}_{n}$ was done in [3] and it is nicely explained in [5]. The following statement is a consequence of the above mentioned work [3], and will be used often in our later arguments.

Proposition 2.1. Let $q$ be a prime and let $n$ be a natural number with q-adic expansion $n=\sum_{j=0}^{k} b_{j} q^{j}$. Let $\lambda$ be a partition of $n$. If $q$ does not divide $\chi^{\lambda}(1)$, then $\left|\mathcal{H}^{q^{k}}(\lambda)\right|=b_{k}$ and $\left|C_{q}(\lambda)\right|=b_{0}$. 
We conclude this section by recalling a few facts on the irreducible characters of $\mathfrak{A}_{n}$. Given a partition $\lambda$ of $n$, we denote by $\lambda^{\prime}$ its conjugate partition. When $\lambda \neq \lambda^{\prime}$, then $\left(\chi^{\lambda}\right)_{\mathfrak{A}_{n}}=\left(\chi^{\lambda^{\prime}}\right)_{\mathfrak{A}_{n}}$ is an irreducible character of $\mathfrak{A}_{n}$. Otherwise, if $\lambda=\lambda^{\prime}$, then $\left(\chi^{\lambda}\right)_{\mathfrak{A}_{n}}=\phi+\phi^{g}$, where $\phi$ is irreducible and $g \in \mathfrak{S}_{n} \backslash \mathfrak{A}_{n}$. These considerations together with $[6$, Section 4$]$ imply the following proposition.

Proposition 2.2. Let $\phi \in \operatorname{Irr}\left(\mathfrak{A}_{n}\right)$ and let $\chi \in \operatorname{Irr}\left(\mathfrak{S}_{n}\right)$ be such that $\phi$ is a constituent of the restriction $\chi_{\mathfrak{A}_{n}}$. If $\chi$ lies in the principal $p$-block of $\mathfrak{S}_{n}$, then $\phi$ lies in the principal p-block of $\mathfrak{A}_{n}$. Moreover, $\phi(1) \in\{\chi(1), \chi(1) / 2\}$.

3. Theorem A. In this section, we focus on proving Theorem A of the introduction. We start by fixing some notation, that will be kept for the rest of the article. Let $n=\sum_{j=0}^{k} b_{j} q^{j}$ be the $q$-adic expansion of $n$. Moreover we let $a \in\{0,1, \ldots, p-1\}$ and $w \in \mathbb{N}$ be such that $n=a+p w$. We let $\mathcal{B}_{n}(p, q)$ be the set of partitions of $n$ labelling irreducible characters in the principal $p$-block of $\mathfrak{S}_{n}$ and having degree divisible by $q$.

Theorem A of the introduction follows from the combination of Theorem 1.1 with the following statement.

Theorem 3.1. Let $n \geq 5$, and let $p$ and $q$ be distinct primes with $p<q \leq n$. Let $B_{0}$ be the principal p-block of either $\mathfrak{S}_{n}$ or $\mathfrak{A}_{n}$. Then $B_{0}$ contains an irreducible character $\chi$ of degree divisible by $q$.

Proof. Our first aim is to show that $\mathcal{B}_{n}(p, q) \neq \emptyset$. This will prove the statement of Theorem 3.1 for $\mathfrak{S}_{n}$. For technical reasons, we start by assuming that the following holds:

$$
n \neq\left(b_{k}+1\right) q^{k}-a-1 .
$$

In this situation, we split our argument into two cases.

Case 1. Suppose that $q^{k} \nmid p w$. We first assume also that $b_{k} q^{k}-a-1 \geq a+1$ and we choose $\lambda$ to be the following partition of $n$ :

$$
\lambda=\left(b_{k} q^{k}-a-1, a+1,1^{n-b_{k} q^{k}}\right) .
$$

We start by observing that $\lambda$ is a well defined partition as $b_{k} q^{k}-a-1 \geq a+1$. We notice that $h_{1,1}(\lambda)=p w$ and therefore that $C_{p}(\lambda)=(a)$. Hence $\chi^{\lambda}$ lies in the principal $p$-block of $\mathfrak{S}_{n}$. Since $q^{k} \nmid h_{1,1}(\lambda)$ and since

$$
h_{1, \lambda_{1}}(\lambda)<\cdots<h_{1,3}(\lambda)<h_{1,2}(\lambda)=b_{k} q^{k}-a-1<b_{k} q^{k},
$$

we deduce that $\left|\mathcal{H}_{1}^{q^{k}}(\lambda)\right|<b_{k}$. We now turn to the analysis of hook-lengths in the second row of $\lambda$. We observe that $h_{2,1}(\lambda)=a+1+n-b_{k} q^{k}$ is not divisible by $q^{k}$ thanks to our technical hypothesis $(\star)$. Moreover, $h_{2, c}(\lambda) \leq h_{2,2}=a<p<q$ for all $c \in\{2, \ldots, a+1\}$. Hence $\left|\mathcal{H}_{2}^{q^{k}}(\lambda)\right|=0$. Similarly $\left|\mathcal{H}_{r}^{q^{k}}(\lambda)\right|=0$ for all $r \geq 3$ because $h_{r, 1}(\lambda) \leq h_{3,1}(\lambda)=n-b_{k} q^{k}<q^{k}$ for all $r \geq 3$. We conclude that $\left|\mathcal{H}^{q^{k}}(\lambda)\right|=\left|\mathcal{H}_{1}^{q^{k}}(\lambda)\right|<b_{k}$. It follows that $\lambda \in B_{n}(p, q)$ by Proposition 2.1.

We now assume that $b_{k} q^{k}-a-1<a+1$. In particular, we have that $b_{k} q^{k}<2(a+1) \leq 2 p$. Since $2 \leq p<q$, it follows that $b_{k}=k=1$, that $q \leq 2 a+1$, and that $n=b_{0}+q=a+p w$ for some $w \leq 3$. 
Under this hypothesis, we choose $\lambda$ to be the following partition of $n$.

$$
\lambda= \begin{cases}\left(a, a, 1^{n-2 a}\right) & \text { if } w=1 \\ \left(q-1, a+1,1^{n-q-a}\right) & \text { if } w \geq 2 \text { and } b_{0}<q-1 \\ \left(a+p, \alpha+1,1^{p-\alpha-1}\right) & \text { if } w \geq 2 \text { and } b_{0}=q-1\end{cases}
$$

Here $\alpha$ is defined as $\alpha=(a-1) / 2$. We will show that $\alpha \in \mathbb{N}$ and that $\lambda$ is well defined.

When $w=1$, then $n=a+p=b_{0}+q$ and $\lambda=\left(a, a, 1^{p-a}\right)$. In this case, we notice that $h_{2,1}(\lambda)=p$ and therefore that $C_{p}(\lambda)=(a)$. Hence $\chi^{\lambda}$ lies in the principal $p$-block of $\mathfrak{S}_{n}$. Moreover, $h_{1,1}(\lambda)=p+1$. Since $n \geq 5$, we deduce that $h_{1,1}(\lambda)<q$. It follows that $h_{i, j}(\lambda)<q$ for all nodes $(i, j)$. We conclude that $\mathcal{H}^{q}(\lambda)=\emptyset$. It follows that $\lambda \in B_{n}(p, q)$ by Proposition 2.1.

When $w \geq 2$ and $b_{0}<q-1$, then $n=a+p w=b_{0}+q$ and $\lambda=(q-1, a+$ $\left.1,1^{n-q-a}\right)$. The partition is well defined as $q-1 \geq a+1$ and as $q+a \leq n$. We observe that $h_{1,1}(\lambda)=p w$ and therefore that $C_{p}(\lambda)=(a)$. Hence $\chi^{\lambda}$ lies in the principal $p$-block of $\mathfrak{S}_{n}$. We notice that $h_{1,2}(\lambda)=q-1<q$ and that $h_{2,1}(\lambda)=b_{0}+1<q$. It follows that $\mathcal{H}^{q}(\lambda)=\emptyset$ and that $\lambda \in B_{n}(p, q)$ by Proposition 2.1.

Finally, when $w \geq 2$ and $b_{0}=q-1$, we have $n=a+p w=(q-1)+q$. Since $q \leq 2 a+1$, we deduce that $w=2$ and hence $n=a+2 p=(q-1)+q$. In particular, $a$ is odd. Let $\alpha \in \mathbb{N}$ be such that $a=2 \alpha+1$ and let $\lambda=(a+$ $\left.p, \alpha+1,1^{p-\alpha-1}\right)$. Since $h_{2,1}(\lambda)=h_{1, a+1}(\lambda)=p$, we deduce that $C_{p}(\lambda)=(a)$ and that $\chi^{\lambda}$ lies in the principal $p$-block of $\mathfrak{S}_{n}$. Moreover, $h_{1, \alpha+1}(\lambda)=q+1$ and $h_{1, \alpha+2}(\lambda)=q-1$. This is enough to conclude that $\mathcal{H}^{q}(\lambda)=\emptyset$ and that $\lambda \in B_{n}(p, q)$ by Proposition 2.1.

Case 2. Suppose now that $q^{k} \mid p w$. Then it is easy to see that $n=a+p w=$ $b_{0}+b_{k} q^{k}$, where $a=b_{0}$ and $w>2$. In this case, we consider $\mu$ to be the partition of $n$ defined by

$$
\mu= \begin{cases}(a+p(w-2), p, p) & \text { if } q^{k} \neq 3 \\ (4,2) & \text { if } q^{k}=3 \text { and } a=0 \\ (3,3,1) & \text { if } q^{k}=3 \text { and } a=1 .\end{cases}
$$

First we notice that $\mu$ is well defined as $w>2$. If $q^{k} \neq 3$, then it is easy to see that $C_{p}(\mu)=(a)$ and hence that $\chi^{\mu}$ lies in the principal $p$-block of $\mathfrak{S}_{n}$. We observe that $h_{1, \mu_{1}}(\mu)<\cdots<h_{1,2}(\mu)<h_{1,1}(\mu)=a+p(w-2)+2<b_{k} q^{k}$. It follows that $\left|\mathcal{H}_{1}^{q^{k}}(\mu)\right|<b_{k}$. As to the second and third row, we notice that $h_{2,1}(\mu)=p+1$ is smaller than $q^{k}$ because $q^{k} \neq 3$. Hence $h_{r, c}(\mu)<q^{k}$ for all $r \in\{2,3\}$ and all $c \in\{1, \ldots, p\}$. Hence $\left|\mathcal{H}_{2}^{q^{k}}(\mu)\right|=\left|\mathcal{H}_{3}^{q^{k}}(\mu)\right|=0$ and $\left|\mathcal{H}^{q^{k}}(\mu)\right|<b_{k}$. Therefore $\mu \in \mathcal{B}_{n}(p, q)$ by Proposition 2.1. If $q^{k}=3$, then $p=2=b_{k}$ as $p<q$ and $n \geq 5$. It follows that $n=6$ or that $n=7$. In both these cases, the partition $\mu$ chosen above lies in $\mathcal{B}_{n}(2,3)$. We notice that the partition $\mu$ defined above lies in $\mathcal{B}_{n}(p, q)$ independently from the validity of technical hypothesis $(\star)$.

In order to conclude the proof, we need now to drop the hypothesis $(\star)$. Hence we suppose that $n=\left(b_{k}+1\right) q^{k}-a-1$. We can also assume that $q^{k} \nmid p w$, 
as this case was already settled in the previous paragraph, by choosing the partition $\mu \in \mathcal{B}_{n}(p, q)$. In this last situation, we consider the following partition $\nu$ of $n$ :

$$
\nu= \begin{cases}\left(q^{k}-1, a+1,1^{b_{k} q^{k}-2 a-1}\right) & \text { if } a \neq 0, \\ \left(q^{k}, 1^{b_{k} q^{k}-1}\right) & \text { if } a=0 .\end{cases}
$$

If $a \neq 0$, then $h_{1,1}(\nu)=n-a=p w$. Hence $C_{p}(\nu)=(a)$ and therefore $\chi^{\nu}$ lies in the principal $p$-block of $\mathfrak{S}_{n}$. Since $q^{k} \nmid p w$, we deduce that $q^{k}$ does not divide $h_{1,1}(\nu)$, and we have

$$
h_{1, j}(\nu) \leq h_{1,2}(\nu)=q^{k}-1<q^{k} \quad \text { for all } 2 \leq j \leq \nu_{1} .
$$

It follows that $\mathcal{H}_{1}^{q^{k}}(\nu)=\emptyset$. Similarly $h_{2,1}(\nu)=b_{k} q^{k}-a$ is not divisible by $q^{k}$ and $h_{2, c}(\nu) \leq a<q^{k}$ for all $2 \leq c \leq a+1$. Hence $\mathcal{H}_{2}^{q^{k}}(\nu)=\emptyset$. Finally we observe that $h_{r, 1}(\nu)=b_{k} q^{k}-2 a-(r-2)$ for all $3 \leq r \leq b_{k} q^{k}-2 a+1$. It follows that the first column of $\nu$ admits at most $b_{k}-1$ nodes having corresponding hook of length divisible by $q^{k}$. We conclude that $\left|\mathcal{H}^{q^{k}}(\nu)\right|<b_{k}$, and therefore that $\nu \in \mathcal{B}_{n}(p, q)$ by Proposition 2.1.

If $a=0$, then with a completely similar argument, we see that $\chi^{\nu}$ lies in the principal $p$-block of $\mathfrak{S}_{n}$. We also observe that $\mathcal{H}_{1}^{q^{k}}=\emptyset$ and that $\left|\mathcal{H}^{q^{k}}(\nu)\right|<$ $b_{k}-1$. Again Proposition 2.1 implies that $\nu \in \mathcal{B}_{n}(p, q)$.

We have proved that the statement holds for $\mathfrak{S}_{n}$. In order to address the problem for $\mathfrak{A}_{n}$, it is enough to observe that our hypothesis forces $q$ to be an odd prime. This implies that given an irreducible character $\chi$ of $\mathfrak{S}_{n}$ lying in the principal $p$-block of $\mathfrak{S}_{n}$ and such that $q \mid \chi(1)$, then any irreducible constituent of $\chi_{\mathfrak{A}_{n}}$ lies in the principal $p$-block of $\mathfrak{A}_{n}$ and has degree divisible by $q$ by Proposition 2.2 .

As mentioned at the beginning of the section, Theorem A follows immediately from Theorems 3.1 and 1.1 .

4. Theorem B. We now proceed with the study of the field of values of the irreducible character identified by Theorem A. In particular, we will prove Theorem B of the introduction.

We recall that by $\mathcal{B}_{n}(p, q)$ we mean the set of partitions of $n$ labelling irreducible characters of $\mathfrak{S}_{n}$ lying in the principal $p$-block of $\mathfrak{S}_{n}$ and whose degree is divisible by $q$. Moreover, we denote by $\Omega_{n}(p, q)$ the subset of $\operatorname{Irr}\left(\mathfrak{A}_{n}\right)$ consisting of rational-valued irreducible characters lying in the principal $p$ block of $\mathfrak{A}_{n}$ such that $q$ divides their degrees. We will often use the following lemma in our later arguments.

Lemma 4.1. Let $\lambda \in \mathcal{B}_{n}(p, q)$. If $\lambda \neq \lambda^{\prime}$, then $\left(\chi^{\lambda}\right)_{\mathfrak{A}_{n}} \in \Omega_{n}(p, q)$.

Proof. Since irreducible characters of $\mathfrak{S}_{n}$ have rational values, this is just a straightforward consequence of Proposition 2.2.

We start by dealing with the situation where $p<q$. The proof of the following proposition is partially based on ideas already explained in the proof of Theorem 3.1. 
Proposition 4.2. Let $n \geq 5$, and let $p$ and $q$ be distinct primes with $2 \leq p<$ $q \leq n$. Then the principal p-block of $\mathfrak{A}_{n}$ admits an irreducible character $\phi$ of degree divisible by $q$ such that $\mathbb{Q}[\phi]=\mathbb{Q}$.

Proof. As usual, we let $n=\sum_{j=0}^{k} b_{j} q^{j}$ be the $q$-adic expansion of $n$, and we set $a \in\{0,1, \ldots, p-1\}$ and $w \in \mathbb{N}$ be such that $n=a+p w$. We aim to show that under our current hypothesis, $\Omega_{n}(p, q) \neq \emptyset$.

As done in the proof of Theorem 3.1, we start by assuming that the following holds:

$$
n \neq\left(b_{k}+1\right) q^{k}-a-1 .
$$

We first assume that $q^{k} \nmid p w$ and that $b_{k} q^{k} \geq 2(a+1)$. In this case, we consider

$$
\lambda=\left(b_{k} q^{k}-a-1, a+1,1^{n-b_{k} q^{k}}\right) .
$$

In the proof of Theorem 3.1, we have seen that $\lambda \in \mathcal{B}_{n}(p, q)$. Hence, if $\lambda \neq \lambda^{\prime}$, then $\left(\chi^{\lambda}\right)_{\mathfrak{A}_{n}} \in \Omega_{n}(p, q)$ by Lemma 4.1. We observe that $\lambda \neq \lambda^{\prime}$ unless

(i) $a=0$ and $n=p w=2 q^{k}-3$, or

(ii) $a=1$ and $n=1+p w=2 q^{k}-4$.

In these cases, we consider the partition $\zeta$ of $n$ defined as follows:

$$
\zeta= \begin{cases}\left(q^{k}, 1^{q^{k}-3}\right) & \text { if (i) holds } \\ \left(q^{k}-1,2,1^{q^{k}-5}\right) & \text { if (ii) holds }\end{cases}
$$

In both cases (i) and (ii), we observe that $p \neq 2$ and therefore that $q^{k} \geq 5$. It follows that $\zeta$ is well defined. We also notice that $\zeta \neq \zeta^{\prime}$. Using techniques completely similar to the ones adopted to prove Theorem 3.1, we deduce that $\mathcal{H}^{q^{k}}(\zeta)=\emptyset$ and thus that $\zeta \in \mathcal{B}_{n}(p, q)$ by Proposition 2.1. Using Lemma 4.1, we conclude that $\left(\chi^{\zeta}\right)_{\mathfrak{A}_{n}} \in \Omega_{n}(p, q)$.

We now assume that $q^{k} \nmid p w$ and that $b_{k} q^{k} \leq 2 a+1$. Proceeding exactly as in the proof of Theorem 3.1, we observe that $n=b_{0}+q=a+p w$ and we choose $\lambda$ to be the following partition of $n$ :

$$
\lambda= \begin{cases}\left(a, a, 1^{n-2 a}\right) & \text { if } w=1 \\ \left(q-1, a+1,1^{n-q-a}\right) & \text { if } w \geq 2 \text { and } b_{0}<q-1 \\ \left(a+p, \alpha+1,1^{p-\alpha-1}\right) & \text { if } w \geq 2 \text { and } b_{0}=q-1\end{cases}
$$

where $\alpha=(a-1) / 2$.

Since $q \leq 2 a+1$ and $n \geq 5$, we deduce that $a \geq 2$. Moreover, $a+p-1>p-\alpha$. This is enough to deduce that $\lambda \neq \lambda^{\prime}$ and therefore that $\left(\chi^{\lambda}\right)_{\mathfrak{A}_{n}} \in \Omega_{n}(p, q)$ by Lemma 4.1.

Let us now suppose that $q^{k} \mid p w$. This forces $n=a+p w=b_{0}+b_{k} q^{k}$, where necessarily $a=b_{0}$. We let $\mu$ be the partition of $n$ defined by 


$$
\mu= \begin{cases}(a+p(w-2), p, p) & \text { if } q^{k} \neq 3, \\ (4,2) & \text { if } q^{k}=3 \text { and } a=0 \\ (3,3,1) & \text { if } q^{k}=3 \text { and } a=1\end{cases}
$$

It is very easy to check that $\mu \neq \mu^{\prime}$. From the proof of Theorem 3.1, we know that $\mu \in \mathcal{B}_{n}(p, q)$. Hence we conclude that $\left(\chi^{\mu}\right)_{\mathfrak{A}_{n}} \in \Omega_{n}(p, q)$ by Lemma 4.1.

To conclude the proof, we now drop the hypothesis $(\star)$, and we analyze the case where $n=\left(b_{k}+1\right) q^{k}-a-1$. Let $\nu$ be the partition of $n$ defined as follows:

$$
\nu= \begin{cases}\left(q^{k}-1, a+1,1^{b_{k} q^{k}-2 a-1}\right) & \text { if } a \neq 0, \\ \left(q^{k}, 1^{b_{k} q^{k}-1}\right) & \text { if } a=0 .\end{cases}
$$

As shown in the proof of Theorem 3.1, we have that $\nu \in \mathcal{B}_{n}(p, q)$. Moreover, it is not difficult to check that $\nu \neq \nu^{\prime}$ unless

(i) $a=0$ and $b_{k}=1$, or

(ii) $a=1$ and $b_{k}=1$.

Outside cases (i) and (ii), we have that $\chi^{\nu} \in \Omega_{n}(p, q)$ by Lemma 4.1.

In case (i), we have that $n=p w=2 q^{k}-1$ and in particular $p \neq 2$. Then we consider

$$
\delta= \begin{cases}\left(q^{k}-p, 3,2^{p-2}, 1^{q^{k}-p}\right) & \text { if } q^{k}>p+3 \\ (6,2,1) & \text { otherwise }\end{cases}
$$

The partition $\delta$ is well defined as $q^{k} \leq p+3$ implies that $n=9, p=3$, and $q=5$. In this case, it is easy to check that $(6,2,1) \in \mathcal{B}_{9}(3,5)$ and that $\chi^{(6,2,1)} \in \Omega_{9}(3,5)$.

If $q^{k}>3$, then $h_{1,1}(\delta)=p(w-1)$ and $h_{2,2}(\delta)=p$. This implies that $C_{p}(\delta)=\emptyset$ and therefore that $\chi^{\delta}$ lies in the principal $p$-block of $\mathfrak{S}_{n}$. We also have that $q^{k}$ does not divide $h_{1,1}(\delta)$ and $h_{2,2}(\delta)$. Moreover, we observe that $h_{1,2}(\delta)=q^{k}-2, h_{2,1}(\delta)=q^{k}+1$, and $h_{3,1}(\delta)=q^{k}-1$. This is enough to deduce that $\left|\mathcal{H}^{q^{k}}(\delta)\right|=0$, and therefore to conclude that $\delta \in \mathcal{B}_{n}(p, q)$ by Proposition 2.1. Since $\delta \neq \delta^{\prime}$, Lemma 4.1 implies that $\left(\chi^{\delta}\right)_{\mathfrak{A}_{n}} \in \Omega_{n}(p, q)$.

In case (ii), we have that $n=p w+1=2 q^{k}-2$, hence again $p \neq 2$. We consider $\gamma=\left(q^{k}-3,2,1^{q^{k}-1}\right)$. Exactly as before, we verify that $\gamma \in \mathcal{B}_{n}(p . q)$ and that $\gamma \neq \gamma^{\prime}$. This implies that $\left(\chi^{\gamma}\right)_{\mathfrak{A}_{n}} \in \Omega_{n}(p, q)$ by Lemma 4.1.

We now turn to analyze the case where $q<p$. In order to do this, we need to introduce the following notation. Given $n=a+p w=\sum_{i=0}^{k} b_{k} q^{k}$ as above, we let $a=\sum_{i=0}^{k} a_{k} q^{k}$ be the $q$-adic expansion of $a$.

Proposition 4.3. Let $n \geq 5$, and let $p$ and $q$ be distinct primes with $2 \leq q<$ $p \leq n$. Then the principal p-block of $\mathfrak{A}_{n}$ admits an irreducible character $\phi$ of degree divisible by $q$ such that $\mathbb{Q}[\phi]=\mathbb{Q}$ unless one of the following holds:

(i) $n=p=2 q-1$, or $($ ii) $n=8, p=7$, and $q=3$, or (iii) $n=6, p=5$, and $q=2$. 
Proof. We start by assuming that $a=0$. In this case, pick $\lambda=\left(b_{k} q^{k}, 1^{n-b_{k}} q^{k}\right)$ and we have that $\lambda \in \mathcal{B}_{n}(p, q)$ by [1, Lemma 3.7]. Moreover, a simple calculation shows that if $n \neq 2 b_{k} q^{k}-1$, then $\lambda \neq \lambda^{\prime}$ and hence $\left(\chi^{\lambda}\right)_{\mathfrak{A}_{n}} \in \Omega_{n}(p, q)$ by Lemma 4.1. On the other hand, when $n=2 b_{k} q^{k}-1$, then necessarily $w \neq 2, b_{k}=1$ and hence $n=p w=2 q^{k}-1$. In this setting, we choose $\mu=$ $\left(n-(q-1), 1^{q-1}\right)$. It is easy to see that $C_{p}(\mu)=\emptyset$ and that $C_{q}(\mu)=\left(q, 1^{q-1}\right)$. Hence, $\chi^{\mu}$ lies in the principal $p$-block of $\mathfrak{S}_{n}$ and $\left|C_{q}(\mu)\right|=2 q-1>b_{0}$. It follows that $\mu \in \mathcal{B}_{n}(p, q)$ by Proposition 2.1. Moreover $\mu \neq \mu^{\prime}$ unless $k=1$. Hence using Lemma 4.1, we conclude that $\left(\chi^{\mu}\right)_{\mathfrak{A}_{n}} \in \Omega_{n}(p, q)$ unless $n=p=2 q-1$. In this latter case, it is not possible to find an irreducible character of the principal $p$-block of $\mathfrak{A}_{n}$, whose degree is divisible by $q$. Suppose for a contradiction that such a character $\phi$ exists and let $\lambda$ be a partition of $n$ such that $\phi \mid\left(\chi^{\lambda}\right)_{\mathfrak{A}_{n}}$. Then $C_{p}(\lambda)=\emptyset$ and hence $\lambda=\left(n-x, 1^{x}\right)$ for some $1 \leq x \leq n-2$. Since $q$ divides $\phi(1)$, we have that $q$ divides $\chi^{\lambda}(1)$. It is well known that $\chi^{\left(n-x, 1^{x}\right)}(1)=\left(\begin{array}{c}n-1 \\ x\end{array}\right)$. It follows that $q$ divides $\chi^{\lambda}(1)$ if and only if $x=q-1$. Hence $\lambda=\left(q, 1^{q-1}\right)=\lambda^{\prime}$ and $\mathbb{Q}[\phi]=\mathbb{Q}[\sqrt{p}]$ by $[2,2.5 .13]$.

We now turn to study the case where $1 \leq a \leq p-1$.

If $p w>b_{k} q^{k}$, then we consider $\mu$ to be the partition of $n$ defined by

$$
\mu= \begin{cases}\left(b_{k} q^{k}-1, a+1,1^{n-\left(b_{k} q^{k}+a\right)}\right) & \text { if } n \neq\left(b_{k}+1\right) q^{k}-1, \\ \left(a, 2,1^{p-2}\right) & \text { if } n=\left(b_{k}+1\right) q^{k}-1, q=2, w=1, \\ & \text { and } a=p-3, \\ \left(b_{k} q^{k}-2, a+1,1^{n-\left(b_{k} q^{k}+a-1\right)}\right) & \text { otherwise. }\end{cases}
$$

From [1, Lemma 3.9], we know that $\mu \in \mathcal{B}_{n}(p, q)$. It is easy to check that $\mu=\mu^{\prime}$ if and only if

(i) $n=1+p w=2 q^{k}-2$, or

(ii) $n=8, p=7$, and $q=3$.

In all other cases, $\left(\chi^{\mu}\right)_{\mathfrak{A}_{n}} \in \Omega_{n}(p, q)$ by Lemma 4.1.

If $n=1+p w=2 q^{k}-2$, then we pick $\rho=\left(q^{k}-3,2,1^{q^{k}-1}\right)$. If $q^{k} \neq 4$, then $\rho$ is well defined. We observe that $C_{p}(\rho)=(1)$ and that $C_{q^{k}}(\rho)=\rho$. Using Proposition 2.1, we deduce that $\rho \in \mathcal{B}_{n}(p, q)$. Using Lemma 4.1, we conclude that $\left(\chi^{\rho}\right)_{\mathfrak{A}_{n}} \in \Omega_{n}(p, q)$ as we clearly have that $\rho \neq \rho^{\prime}$.

On the other hand, if $q^{k}=4$, then $n=6$ and $p=5$. In this case, we have $\Omega_{6}(5,2)=\emptyset$. Similarly, if $n=8, p=7$, and $q=3$, then direct verification shows that $\Omega_{8}(7,3)=\emptyset$.

Finally, if $p w<b_{k} q^{k}$, then we let $\omega$ be the partition of $n$ defined by

$$
\omega= \begin{cases}\left(a, a-a_{k} q^{k}+1,1^{n-2 a+a_{k} q^{k}-1}\right) & \text { if } a_{k}>0, \\ (n-2,2) & \text { if } a_{k}=0 \text { and } a=1, \\ \left(a, 1^{w p}\right) & \text { if } a_{k}=0 \text { and } a>1 \text { and } n \neq b_{k} q^{k}, \\ \left(a, 2,1^{w p-2}\right) & \text { if } a_{k}=0 \text { and } a>1 \text { and } n=b_{k} q^{k} .\end{cases}
$$


By [1, Lemma 3.11], we know that $\omega \in \mathcal{B}_{n}(p, q)$. Moreover, it is easy to check that $\omega \neq \omega^{\prime}$. Hence $\left(\chi^{\omega}\right)_{\mathfrak{A}_{n}} \in \Omega_{n}(p, q)$ by Lemma 4.1 .

Theorem B is a direct consequence of Propositions 4.2 and 4.3.

Funding. Open access funding provided by Universitá degli Studi di Firenze within the CRUI-CARE Agreement.

Open Access. This article is licensed under a Creative Commons Attribution 4.0 International License, which permits use, sharing, adaptation, distribution and reproduction in any medium or format, as long as you give appropriate credit to the original author(s) and the source, provide a link to the Creative Commons licence, and indicate if changes were made. The images or other third party material in this article are included in the article's Creative Commons licence, unless indicated otherwise in a credit line to the material. If material is not included in the article's Creative Commons licence and your intended use is not permitted by statutory regulation or exceeds the permitted use, you will need to obtain permission directly from the copyright holder. To view a copy of this licence, visit http://creativecommons. org/licenses/by/4.0/.

Publisher's Note Springer Nature remains neutral with regard to jurisdictional claims in published maps and institutional affiliations.

\section{References}

[1] Giannelli, E., Malle, G., Vallejo, C.: Even degree characters in principal blocks. J. Pure Appl. Algebra 223(2), 900-907 (2019)

[2] James, G., Kerber, A.: The Representation Theory of the Symmetric Group. Encyclopedia of Mathematics and its Applications, vol. 16. Addison-Wesley Publishing Co., Reading, MA (1981)

[3] Macdonald, I.G.: On the degrees of the irreducible representations of symmetric groups. Bull. Lond. Math. Soc. 3, 189-192 (1971)

[4] Malle, G., Navarro, G.: Brauer's height zero conjecture for two primes. Math. Z. 295(3-4), 1723-1732 (2020)

[5] Olsson, J.B.: p-blocks of symmetric and alternating groups and their covering groups. J. Algebra 128(1), 188-213 (1990)

[6] Olsson, J.B.: Combinatorics and Representations of Finite Groups. Vorlesungen aus dem Fachbereich Mathematik der Universität Essen, Heft 20 (1993)

Eugenio Giannelli and Elena Meini

Dipartimento di Matematica e Informatica Ulisse Dini

Universita degli Studi di Firenze

Florence

Italy

e-mail: eugeniogiannelli4@gmail.com

Elena Meini

e-mail: elena.meini@stud.unifi.it 
Received: 2 May 2020

Accepted: 27 September 2020. 